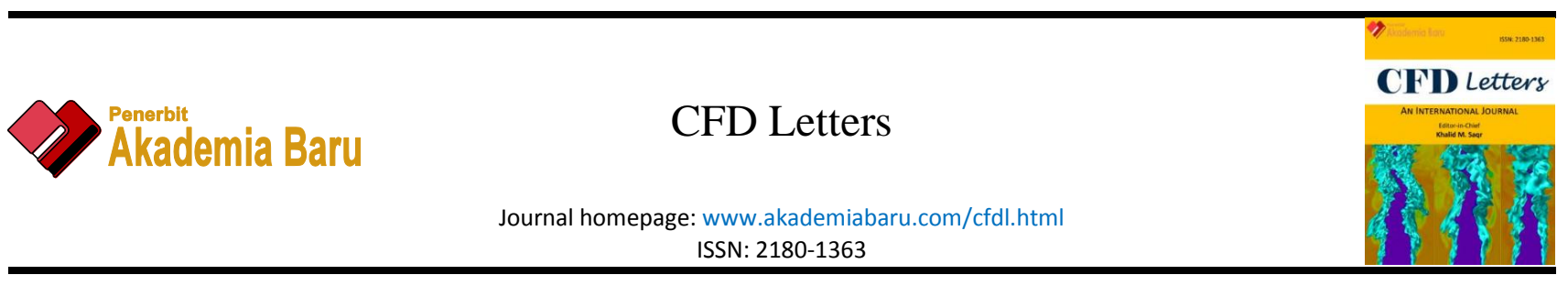

\title{
CFD Analysis of Slipstreaming and Side Drafting Techniques Concerning Aerodynamic Drag in NASCAR Racing
}

\author{
Edwin Chern Junn Gan ${ }^{1,}{ }^{*}$, Mikhail Fong ${ }^{1}$, Yee Luon $\mathrm{Ng}^{1}$ \\ 1 Department of Mechanical Engineering, College of Engineering, Universiti Tenaga Nasional, Putrajaya Campus, 43000 Kajang, Selangor, \\ Malaysia
}

\section{ARTICLE INFO}

Article history:

Received 20 May 2020

Received in revised form 19 July 2020

Accepted 24 July 2020

Available online 30 July 2020

\section{ABSTRACT}

NASCAR has given enormous concentration on aerodynamics in racing in which drivers rely solely on aerodynamic techniques in pursuit of podium finishes. Since drag accounts for roughly $90 \%$ total resistance at top speed, NASCAR drivers have resorted to such techniques in gaining aerodynamic advantages. Presently, there are little indepth investigations found on slipstreaming in applications of high-speed NASCAR race cars and even lesser to none on side drafting. The effects of slipstreaming were studied by varying the vehicle spacing and platoon population of platoon members, whereas the effects of side drafting were studied by varying the longitudinal position and lateral separation between trailing and leading vehicles. The results prove that slipstreaming is beneficial to platoon members in significantly reducing aerodynamic drag at short vehicle spacings. However, there exists a 'drag bubble' within specific ranges of vehicle spacing where trailing vehicles experience significant drag increments. Increasing the platoon population is found to decrease the average drag values of platoon members to a certain extent. On the other hand, the side drafting study reveals that the leading car undergoes significant drag increments while the trailing car experiences some drag reduction, thus proving substantial aerodynamic advantages for trailing vehicles in raising their overtaking capabilities.

\section{Keywords:}

Computational fluid dynamics; numerical simulation; stock car racing; drafting; vehicle platoons

Copyright @ 2020 PENERBIT AKADEMIA BARU - All rights reserved

\section{Introduction}

Motor racing has seen the intense use of aerodynamic techniques such as slipstreaming and side drafting in pursuit of aerodynamic advantage at high speeds. Professional racing drivers in NASCAR heavily utilise slipstreaming by forming vehicle platoons and following closely among one another, benefitting themselves with reduced overall drag and higher fuel efficiency. On the other hand, side drafting is also greatly utilised to slow down leading opponents in NASCAR racing. This is extensively used by drivers as they position themselves at the quarter panel of their respective leading opponents and displace large amounts of air from their front fasciae onto their leading opponents'

\footnotetext{
* Corresponding author.

E-mail address: edwingan_cj@hotmail.com (Edwin Chern Junn Gan)
} 
spoilers. This effectively causes their leading opponents to experience significant drag increments and lose speed.

While NASCAR race cars are mechanically able to achieve greater top speeds at wide-open throttle, the use of tapered spacers is imposed to limit their top speeds to roughly $89.4 \mathrm{~m} \mathrm{~s}^{-1}$ (or 200 $\mathrm{mph}$ ) as an initiative to improve the safety of the drivers and spectators at the track. Tapered spacers restrict the air intake of the engine, thus reducing engine power. This reduced power makes it more difficult for the cars to gain speed and forces drivers to resort to aerodynamic techniques in racing, especially since aerodynamic drag accounts for roughly $90 \%$ of the total resistance at top speed [1].

Multiple studies on drag savings obtained from slipstreaming have been conducted using various vehicle types. Zabat et al., [1] and Robertson et al., [2] predicted drag reduction for vehicle platoons by conducting wind tunnel experiments on one-eight scale minivan models and one-twentieth scale commercial lorry models respectively and concluded that vehicle platooning can indeed induce a reduction of drag. It is found that the average drag coefficient of platoon members reduces with the decrease in vehicle separation, with a more rapid reduction occurring at shorter separations [1-2]. For instance, the aforementioned study by Robertson et al., [2] has shown the 8-lorry platoon at spacings of $0.5,1$ and 1.5 lorry lengths experiencing reductions of at least $48 \%, 29 \%$ and $24 \%$ respectively, relative to a lorry driving in isolation.

Also, adding members into platoons results in further reductions of average drag coefficient among platoon members [1-2]. However, findings illustrate that adding more vehicles into an already-long platoon will not create as big an impact to the average drag coefficient as compared to when the platoon was of a shorter length [1-2]. This can be observed in the study by Zabat et al., [1] where the 2-car, 3-car and 4-car platoons experience reductions in average drag coefficient of up to roughly $35 \%, 45 \%$ and $49 \%$ respectively. The same pattern for fuel savings concerning the number of cars in the platoon is found by Michaelian and Browand [3] by monitoring the engine speed, velocity and fuel injector pulse width of individual Buick LeSabre sedans in a platoon. It is found that fuel savings of the 2-car, 3-car and 4-car platoons at $3 \mathrm{~m}$ spacing are $5.5 \%, 7.5 \%$ and $8.5 \%$ respectively.

An interesting point to note is that Zabat et al., [1] and Michaelian and Browand [3] found a strong correlation between the position of a vehicle within a platoon and its drag or fuel consumption. It is found that interior vehicles undergo the least drag and experiences the best fuel savings, followed by the trailing vehicle and leading vehicle; however, this only occurs at relatively short spacings between $3 \mathrm{~m}$ and $6 \mathrm{~m}$. Zabat et al., [1] tested vehicle platooning at much longer spacings and found that the leading vehicle eventually experiences the highest drag followed by every consecutive vehicle downstream of it.

More in-depth wind tunnel tests, field tests and CFD simulations on 2-car platoons in a slipstreaming situation at relatively short spacings by Zabat et al., [1], Hong et al., [4] and Brzustowicz et al., [5] have shown at some point that the trailing car experiences a rise in drag and experiences more drag than the leading car. This is evident in 2-car platoon studies by Zabat et al., [1] at less than 0.35 car lengths, Hong et al., [4] at less than 0.43 car lengths and Brzustowicz et al., [5] between 0.25 to 1 car length. These findings give reason to believe that the designs of each vehicle have caused undesirable aerodynamic characteristics at their specific vehicle spacings [6]. This effect is referred to as the 'drag bubble' by drivers and is evident on the track when the trailing car begins slowing down while the leading car accelerates away within this range of separation [6]. This effect is addressed by Jacuzzi and Granlund [6] in reducing these peaks in drag.

Concerning other applications of slipstreaming, multiple studies have been conducted on cyclists riding in two-rider formations in various cycling positions. Belloli et al., [7] studied the effects of drafting and compared findings with other studies by Barry et al., [8], Blocken et al., [9], Edwards and Byrnes [10] and Kyle [11] that analysed various cycling positions (upright, dropped and time-trial 
positions) using various methods (field testing, wind tunnel testing and CFD simulation). A similar trend is found in the aforementioned studies [7-11] in which drag reduction experienced by a trailing cyclist increases with the decrease in its distance from the leading cyclist. Blocken et al., [9] simulated two drafting cyclists using CFD and found that the leading cyclist not only reduces the overpressure in front of the trailing cyclist but also reduces the underpressure at the rear of the latter, consequently reducing the drag experienced by the trailing cyclist. Also, the trailing cyclist reduces the underpressure at the rear of the leading cyclist by filling up the vacuum behind the latter, hence reducing the drag for the leading cyclist [9].

As far as turbulence models for automotive flow problems are concerned, the Reynolds-averaged Navier-Stokes (RANS) approach is the most commonly used due to its computational efficiency, even though other turbulence models like the Large Eddy Simulation (LES) and the Detached Eddy Simulation (DES) are more accurate and robust in predicting turbulence characteristics [12]. Ashton et al., [13] assessed the RANS and DES (a hybrid RANS-LES approach) for use in realistic automotive models and found that the DES approach offers a clear advantage over the RANS models in terms of force coefficients and general flow fields; however, inaccuracies are exhibited even at the finest mesh level [13].

Although there exist numerous studies on slipstreaming in applications of passenger and commercial vehicles, only a limited number of in-depth investigations can be found on slipstreaming in applications of high-speed NASCAR race cars, and presently there exists little or no study at all on side drafting. This study aims to fill that gap and in turn, obtain the best racing strategies based on car placements in both slipstreaming and side drafting techniques with regard to potential drag reduction and overtaking capabilities respectively.

\section{Methodology}

\subsection{Parameters}

The Monster Energy NASCAR Cup Series race held at the Talladega Superspeedway in the autumn of 2019 is taken into consideration as a locational parameter of this study due to the intense use of aerodynamic techniques among drivers.

This study has analysed 2019 NASCAR Gen-6 race cars cruising at a constant velocity of $89.4 \mathrm{~m} \mathrm{~s}$ ${ }^{1}$ (or $200 \mathrm{mph}$ ). Since race organisers terminate races on rainy days to improve track safety, this study has idealised the track conditions to be dry at a constant temperature of $24^{\circ} \mathrm{C}$ conforming to weather forecasts on race day. These factors are considered in obtaining the air properties [14] at the track, as summarised in Table 1.

To determine the flow type of the air, the Reynolds number is calculated using the air properties [14] in Table 1. Where the characteristic length, $L_{C}$ is taken as the length of the car, the Reynolds Number is calculated to be $2.88 \mathrm{exp}+7$ which indicates the flow type around the car to be turbulent. All constant values including air properties used in this study are summarised in Table 1.

\section{Table 1}

Summary of fixed values

\begin{tabular}{ll}
\hline Velocity, $v\left(\mathrm{~m} \mathrm{~s}^{-1}\right)$ & 89.4 \\
Characteristic Length, $L_{C}(\mathrm{~m})$ & 5.036 \\
Density of Air at $24^{\circ} \mathrm{C}, \rho\left(\mathrm{kg} \mathrm{m}^{-3}\right)$ & 1.184 \\
Dynamic Viscosity of Air at $24^{\circ} \mathrm{C}, \mu\left(\mathrm{Ns} \mathrm{m}^{-2}\right)$ & $1.85 \exp -5$ \\
Reynolds Number, $R e$ & $2.88 \exp +7$ \\
\hline
\end{tabular}




\subsection{Car Modelling}

The model used in this study (see Figure 1) is simplified based on the 2019 Chevrolet Camaro Gen-6 NASCAR race car to focus on the flow around the car, especially the frontal and wake regions. Also, a simplified model increases computational efficiency during CFD simulations, providing more opportunity to analyse more configurations.

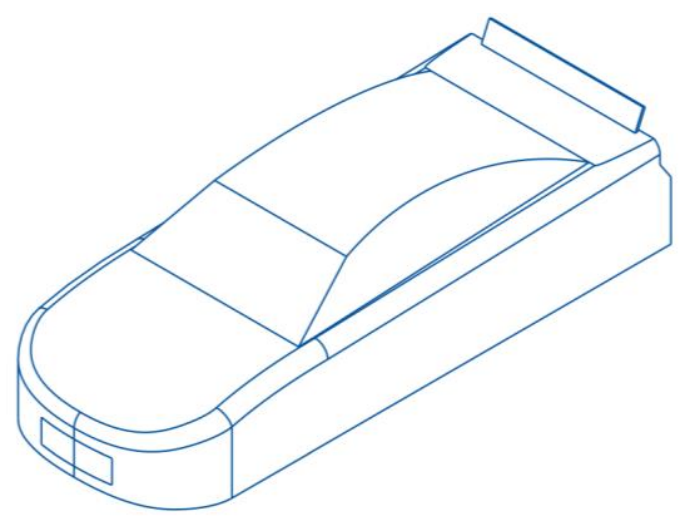

Fig. 1. Idealised model of Gen-6 NASCAR in isometric view

The notable dimensions of the car and spoiler in Table 2 are based on the actual dimensions of the 2019 Monster Energy NASCAR Cup Series race cars conforming to the 2019 rules package by NASCAR.

\begin{tabular}{|c|c|}
\hline Length of car $(\mathrm{m})$ & \\
\hline Width of car (m) & 1.956 \\
\hline Length of spoiler (m) & 1.549 \\
\hline Width of spoiler (m) & 0.203 \\
\hline
\end{tabular}

\subsection{CFD Modelling and Meshing}

This study has employed the ANSYS FLUENT 2019 R2 for CFD simulations. The computational domain is designed to be a free-flow test environment with a blockage ratio of $3.8 \%$ for an isolated car model. While blockage ratios lower than $5 \%$ are generally accepted for aerodynamic analyses of cars in wind tunnel tests [15-16], Choi and Kwon [17] analysed aerodynamic behaviours of bluff bodies with regard to various blockage ratios and concluded that the ratio may be increased to $10 \%$ without having any notable reduction in the accuracy of test results. Therefore, any blockage errors in this current study can safely be neglected. The velocity inlet is placed $5 \mathrm{~m}$ (roughly a car length) in front of the leading car, whereas the pressure outlet is placed $10 \mathrm{~m}$ (roughly 2 car lengths) behind the last trailing car. The top and side boundaries are placed $5 \mathrm{~m}$ and $4 \mathrm{~m}$ (roughly 2 car widths) away from the cars respectively. Since lift results are not considered in this study, the air under the car is omitted to mainly focus on drag reductions due to air blockage among platoon members.

The fluid domain is split into two enclosures (see Figure 2), where the inner enclosure is used as a body of influence with an increased mesh resolution. This numerical model follows simulation 
techniques and best practices of Gan and Salim [18-19]. The more critical air space surrounding the car and the wake region is programmed to utilise finer tetrahedral meshes, whereas the remaining air in the domain is filled up with coarser hex meshes. Additionally, an inflation layer with a first aspect ratio of 5 and a growth rate of $20 \%$ extruding 5 layers is utilised in this study which conforms to best practice guidelines for exterior automotive aerodynamics by Lanfrit [20]. Mesh manipulation in this manner is effective in reducing the node and element count while maintaining mesh resolution and numerical accuracy at the area of interest, thus increasing overall computational efficiency. Also, in slipstreaming configurations that are symmetrical at the longitudinal plane, the simulation utilised half models to further reduce computational costs.

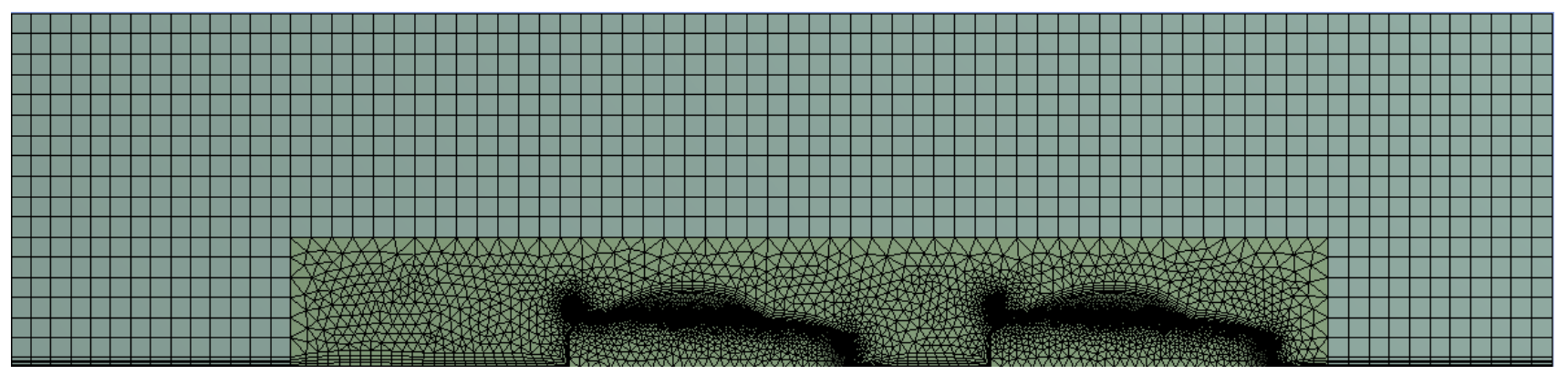

Fig. 2. Mesh model

The velocity inlet is set at a constant velocity of $89.4 \mathrm{~m} \mathrm{~s}^{-1}$ normal to the boundary to simulate NASCAR race cars cruising at top speed with turbulent intensity and turbulent viscosity ratio set at $5 \%$ and 10 respectively, conforming to recommendations by ANSYS [21] for external flows. The pressure outlet is set at atmospheric pressure. The NASCAR geometries and road are defined as walls in non-slip condition, whereas the top, side and symmetry boundaries of the enclosure are of symmetry type. The same boundary conditions apply to all configurations in this study as illustrated in Figure 3.
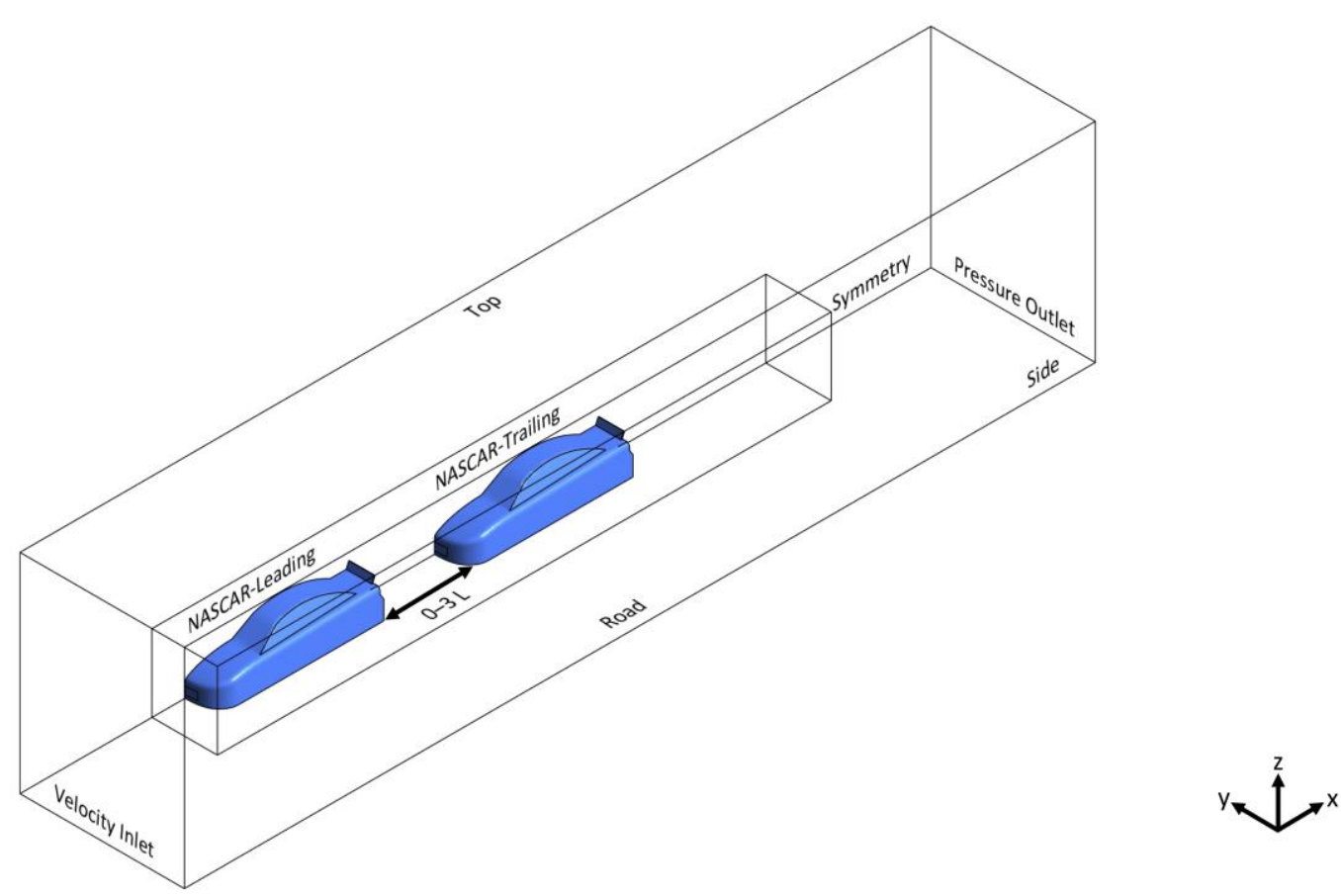

Fig. 3. Boundary conditions. $L=$ Car Length 


\subsection{Numerical Modelling}

Deeper analysis of the RANS model by Fu et al., [12] has shown that variations of the $k-\varepsilon$ model have a clear advantage over the $k-\omega$ model in terms of force and moment coefficients. While Fluent recommends the use of the realizable $k-\varepsilon$ (RKE) for external aerodynamic studies [20], other variations of the RANS model have been employed for automotive flow problems, such as the standard $k-\varepsilon$ (SKE) employed by Li et al., [22] in the study of vehicle movement on pollutant dispersion in urban streets and Blocken et al., [9] in the aerodynamic analysis of two drafting cyclists, as well as the shear-stress transport (SST) $k-\omega$ employed by Jacuzzi and Granlund [6] in the study of passive flow control for drag reduction of the trailing car in a platoon of two NASCAR Xfinity Series race vehicles and Shaharuddin et al., [23] in the flow simulations of generic vehicle models.

The steady-state RANS standard $k-\varepsilon$ (SKE) model with standard wall functions is employed in the current study due to its modest computational demands and wide validation in engineering flow problems [24]. It is worth noting that the racing community employs the RANS turbulence model as a first approximation tool due to its computational efficiency and cost-effectiveness [12]. The convergence criterion for all configurations is set at 1exp-3 [21]. The solution methods employed are summarised in Table 3 and are conformed to best practice guidelines by Lanfrit [20].

Table 3

Solution methods

\begin{tabular}{ll}
\hline Scheme & SIMPLE \\
Gradient & Least Squares Cell Based \\
Pressure & Second Order \\
Momentum & Second Order Upwind \\
Turbulent Kinetic Energy & Second Order Upwind \\
Turbulent Dissipation Rate & Second Order Upwind \\
\hline
\end{tabular}

\section{Results}

\subsection{Isolated Car}

Drag findings of the isolated car in the present study are benchmarked with results obtained by Fu et al., [12] on a NASCAR Gen-6 Cup race car using the Aerodyn Wind Tunnel. The present findings are evaluated to have very minimal offset from the benchmark as summarised in Table 4.

Table 4

Drag results of the isolated car

\begin{tabular}{llll}
\hline & CFD Findings & Wind Tunnel Results & Percentage Error (\%) \\
\hline Drag Coefficient & 0.3740 & 0.3700 & 1.0837 \\
Drag Force (N) & 4232 & - & - \\
\hline
\end{tabular}

To further evaluate the accuracy of the simulation results, a mesh independence test is performed on the isolated car model as summarised in Figure 4. In pursuit of an optimum balance between accuracy and computational cost, the mesh settings bearing the element count of 692691 is employed for all configurations in this study. 


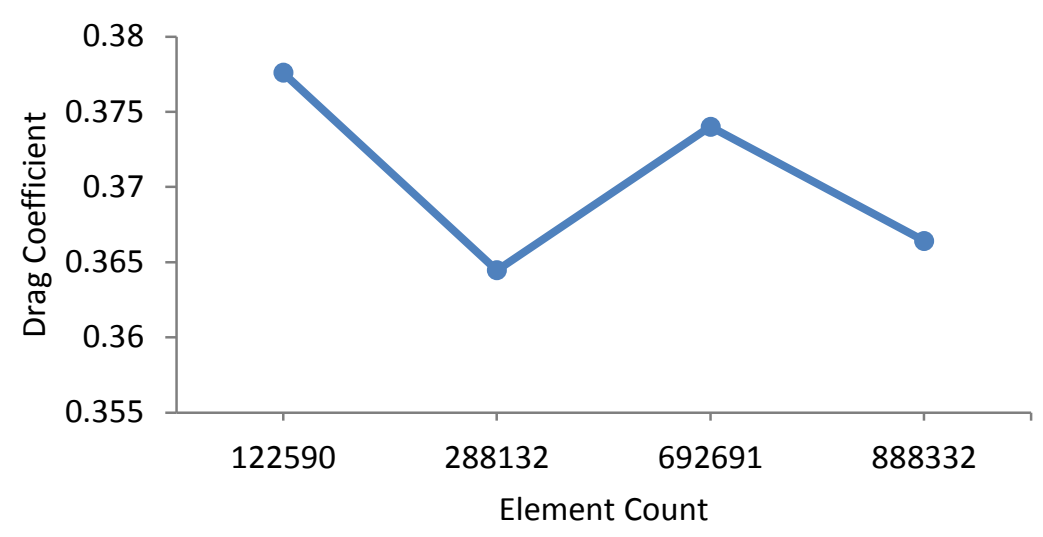

Fig. 4. Mesh independence test of the isolated car model

Streamline plots in Figure 5 illustrate air velocity increasing as air flows over the roof before slowing down drastically in vortices at the recirculation region at the rear of the vehicle. In the same plots, there exists a 'tunnel' of air at lower relative velocity downstream of the car. The consequence of placing trailing cars in that 'tunnel' is of interest in the slipstreaming investigation. Additionally, the contour plot in Figure 5 exhibits higher pressure regions around the front fascia, windshield, spoiler and decklid regions.

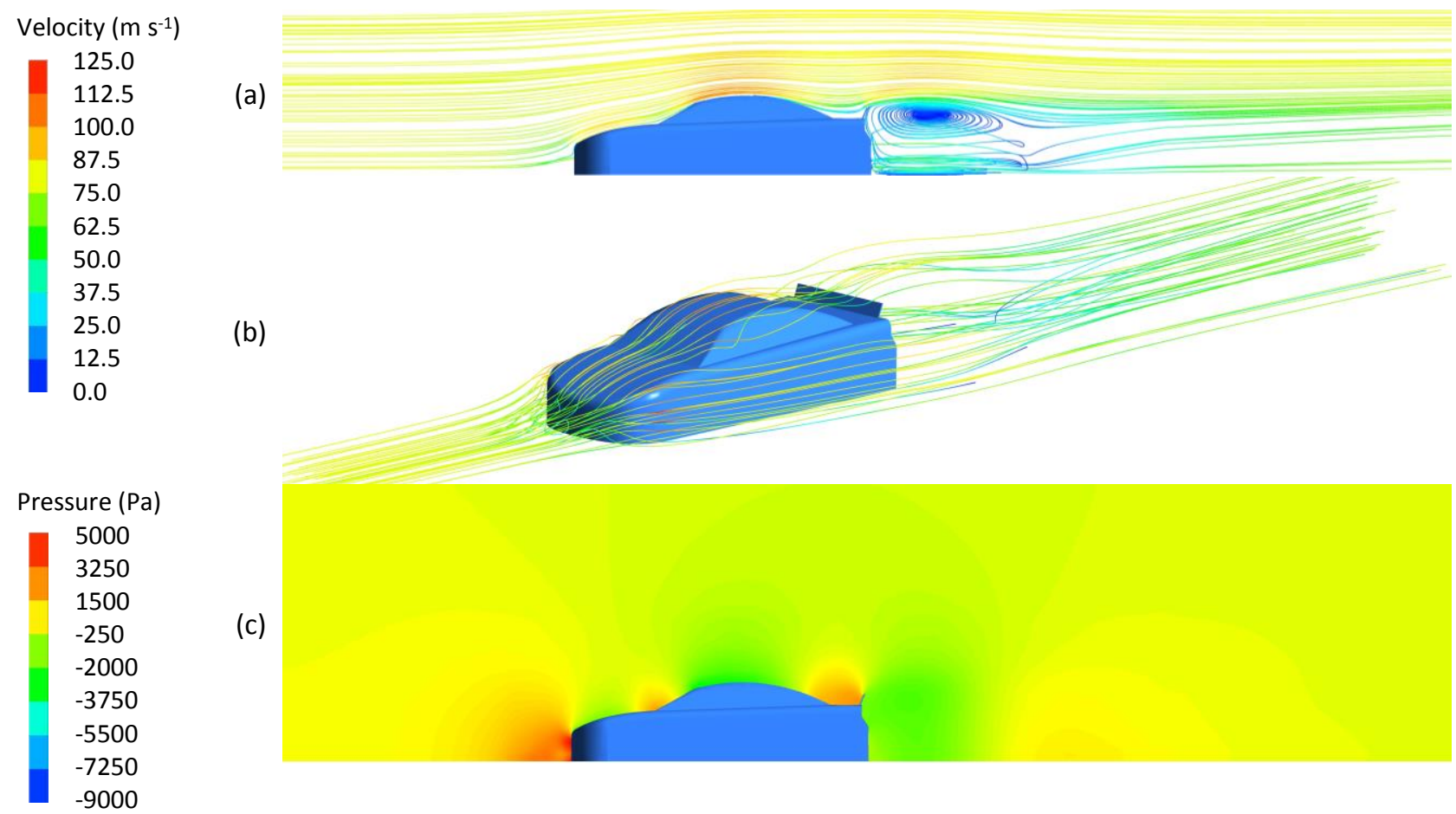

Fig. 5. Isolated car: (a) Longitudinal streamlines of the velocity magnitude, (b) 3D streamlines of the velocity magnitude and $(c)$ contour plot of the pressure magnitude

The wake of the isolated car illustrated by the contour plot in Figure 6 is observed to bear a 'mushroom' shape produced by the counter-rotating upwash similarly found in the wake of a NASCAR Xfinity Series car analysed by Jacuzzi and Granlund [6]. Investigations into generic automotive bodies by Ahmed [25], Vino et al., [26] and Shaharuddin et al., [23], as well as vehicle shapes by Shaharuddin et al., [23] and Pagliarella et al., [27], have also exhibited strong recirculation zones downstream of their respective vehicles with a pair of counter-rotating longitudinal vortices extending in the wake 
region. However, since the model in the current study is an idealised model that is designed to be symmetrical at the longitudinal plane, the contour plot in Figure 6 does not reflect the asymmetrical wake characteristics typically found on accurate NASCAR models with asymmetrical features such as the rear deck fin present on the left side. According to NASCAR, the rear deck fins generate rear side force to prevent cars from going airborne in a spin.
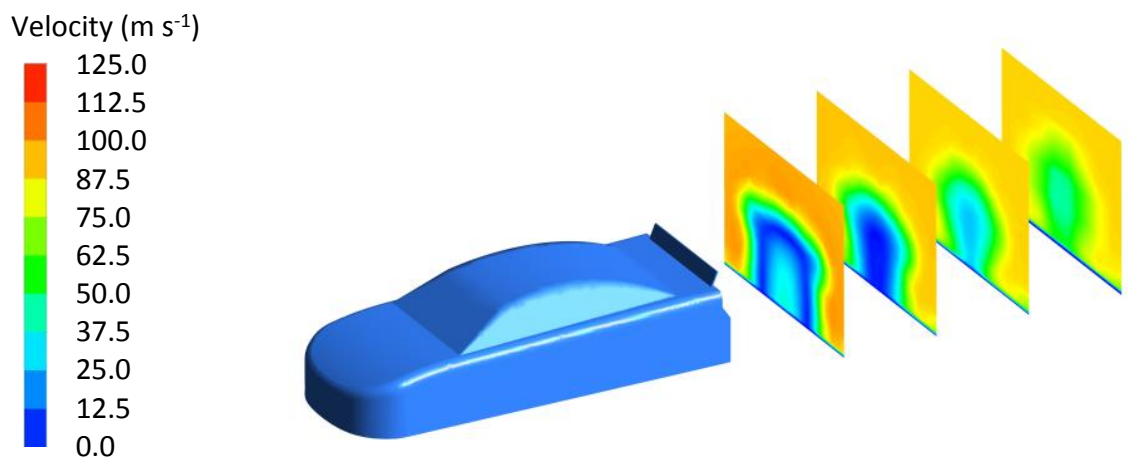

Fig. 6. Contour plot illustrating the wake characteristics of the isolated car at vehicle spacings, $x / L=0.25,0.5,0.75$ and 1 car length

\subsection{Slipstreaming in Various Vehicle Spacings}

The consequence of varying vehicle spacings is studied for potential drag reductions. Figures 7-8 illustrate streamline and contour plots of the velocity and pressure magnitudes respectively of two cars at several vehicle spacings, $x / L$, where $x$ denotes the streamwise separation length between the cars and $L$ denotes the length of one car.

Streamlines in Figure 7 illustrate air blockage from the leading car consequently reducing the velocity of air before reaching the trailing car. Additionally, contour plots in Figure 8 show that the higher-pressure regions around the front fascia, windshield, spoiler and decklid regions are relatively lower on trailing cars. It is observed that at short vehicle spacings, especially at $x / L \leq 0.25$, the trailing car breaches the recirculation region in the wake of the leading car and experiences maximum air blockage. Subsequently, the slipstream of the 2-car platoon behaves similarly to the slipstream of an isolated car at this range of vehicle spacings where there exist very minimal air disturbances between both cars. As vehicle spacing increases, the streamline and contour plots show air velocity and air pressure around the trailing car gradually increasing, where both cars will eventually start having identical velocities and pressures as though they are driving in total isolation from one another.

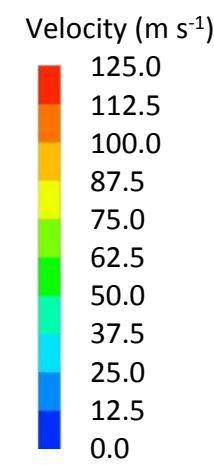

(a)
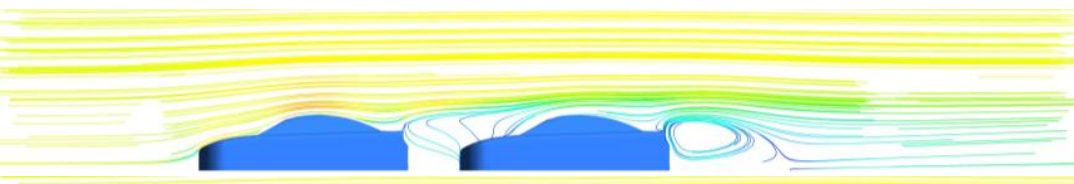

(b)

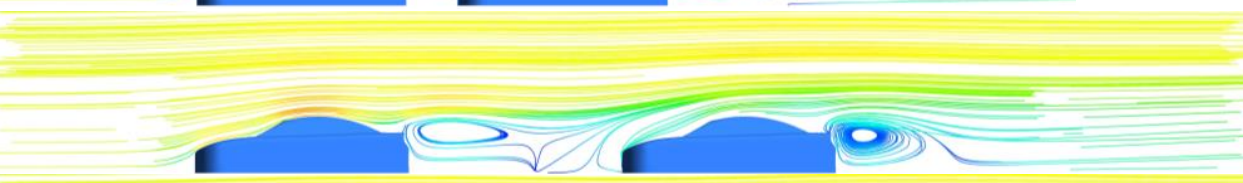

(c)

Fig. 7. Longitudinal streamlines of the velocity magnitude for the 2-car platoon at various vehicle spacings: (a) $x / L=0.25$, (b) $x / L=1$, and (c) $x / L=1.5$ 


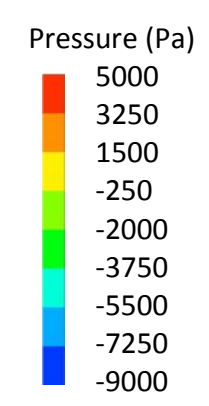

(a)

(b)
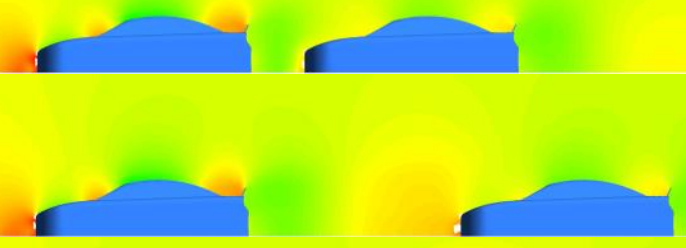

(c)

Fig. 8. Contour plots of the pressure magnitude for the 2 -car platoon at various vehicle spacings: $(a) x / L=$ 0.25 , (b) $x / L=1$, and (c) $x / L=1.5$

Figures 9-11 illustrate the changes in drag ratio with respect to vehicle spacing for different platoon populations. Drag ratio is calculated with Eq. (1), where the drag of individual platoon members is normalised with the drag of the isolated car for easier comparison. Figure 12 illustrates the changes in the average drag ratio of various platoon populations with respect to vehicle spacing. Average drag ratio is calculated with Eq. (2).

Drag Ratio $=\frac{F_{D, \text { member }}}{F_{D, \text { isolated }}}$

Average Drag Ratio $=\frac{\bar{F}_{D, \text { member }}}{F_{D, \text { isolated }}}$

Figures 9-11 show that slipstreaming is most beneficial to a trailing car at short vehicle spacings, $x / L \leq 0.25$, where the trailing car experiences maximum drag reduction. Within vehicle spacings from approximately $x / L=0.25$ to $x / L=1.5$, all trailing members experience significant drag increments with downstream members experiencing lesser drag variations than their upstream members. This drag increment is caused by the 'drag bubble' existing at the wake of the cars as described by drivers and as addressed by Jacuzzi and Granlund [6]. It is interesting to note that the leading car is aerodynamically advantageous in this occurrence where it experiences a reduction in drag and has relatively lesser drag than its trailing member.

Members at the middle of the platoon generally experience the lowest drag at approximate vehicle spacings of $x / L \leq 1.5$. At larger vehicle spacings, the last trailing member has the lowest drag, followed by every consecutive upstream member. Interestingly, it is revealed that there still exists some drag reduction for trailing members even at the largest vehicle spacing, $x / L=3$.

Averaging the drag ratio of the 2-car, 3-car, and 4-car platoons (see Figure 12) reveal maximum slipstreaming benefits at vehicle spacings shorter than $x / L=1$. Vehicle spacing longer than $x / L=1$ car length may still somewhat benefit platoons, but very ineffectively. A similar trend for all curves can be found in a study of drag savings in multiple car platoons by Zabat et al., [1], whereas drag findings by Hong et al., [4] and Brzustowicz et al., [5] show an especially similar trend for 2-car platoon configurations. However, upon comparison with the aforementioned studies [1,4-5], the drag bubble occurs at different vehicle spacings among distinct vehicular shapes and show that the trailing car does not always possess the aerodynamic advantage at small vehicle spacings. 

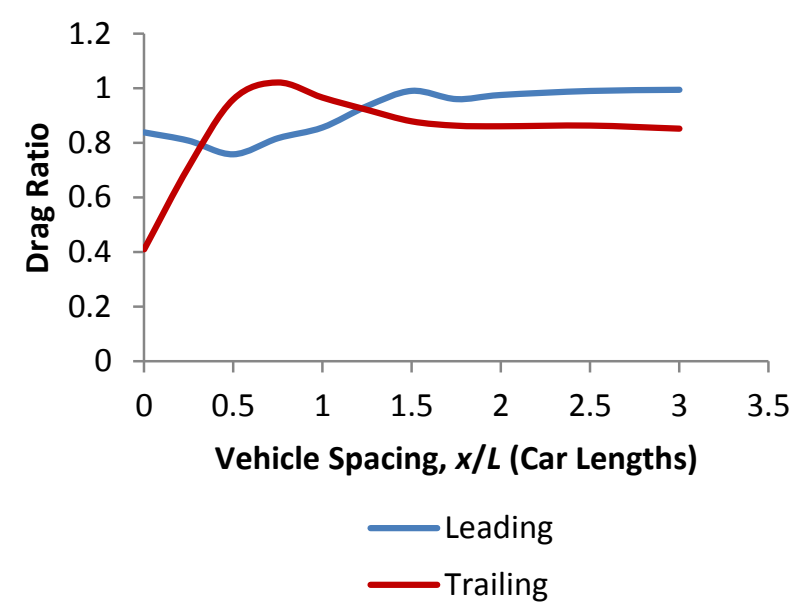

Fig. 9. Drag ratio against vehicle spacing for the 2-car platoon

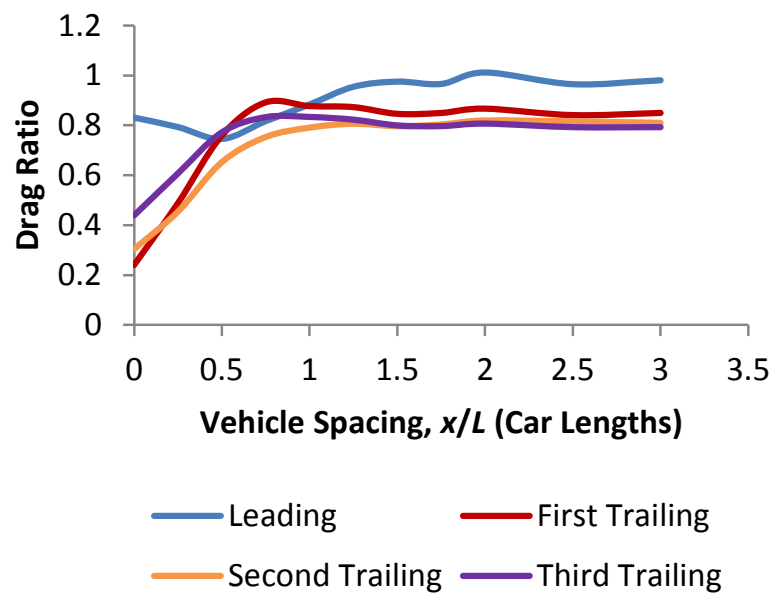

Fig. 11. Drag ratio against vehicle spacing for the 4-car platoon

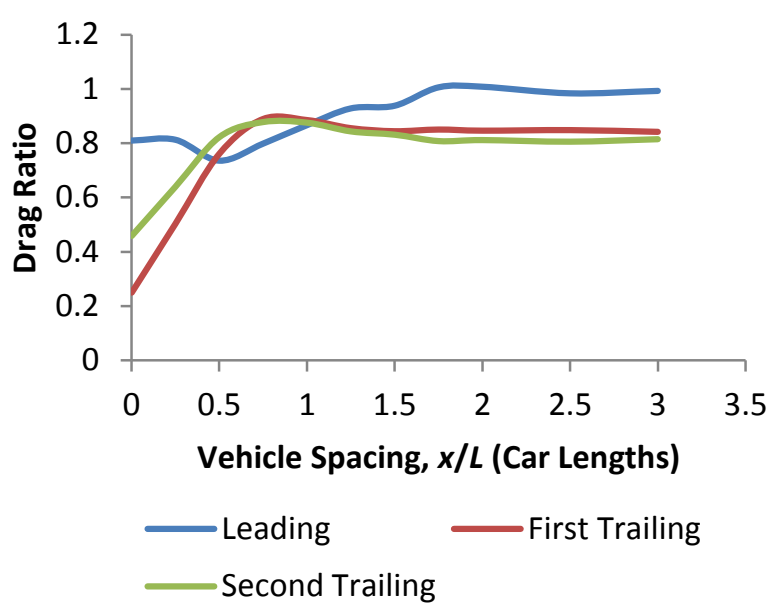

Fig. 10. Drag ratio against vehicle spacing for the 3-car platoon

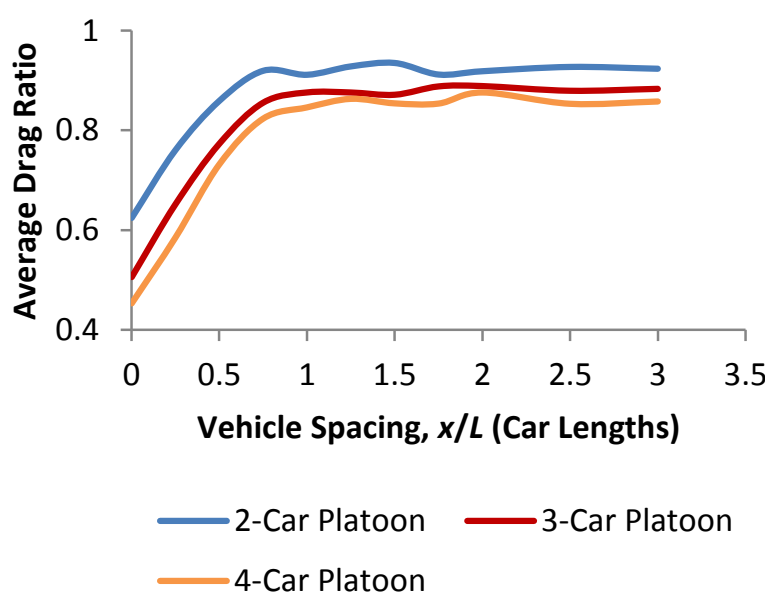

Fig. 12. Average drag ratio against vehicle spacing for the 2-car, 3-car and 4-car platoons

\subsection{Slipstreaming in Various Platoon Populations}

The consequence of placing more members in the platoon is studied for potential drag reductions. Figures 13-14 illustrate streamline and contour plots of the velocity and pressure magnitudes respectively for different platoon populations.

Figures 13-14 show air velocity and air pressure decreasing for every consecutive downstream member. Additionally, the 'tunnel' of air as discussed in the isolated car study is shown in Figure 13 to increase in cross-section as it flows past every downstream member. The same pattern is observed for different platoon populations.

Figure 15 illustrates the changes in average drag ratio with respect to platoon population. It is found that the average drag ratio decreases with the increasing number of platoon members, and will eventually stagnate around a minimum value. This reveals that any drag reduction will be less significant when adding more members in longer platoons. A similar trend can be found in drag findings by Zabat et al., [1]. 


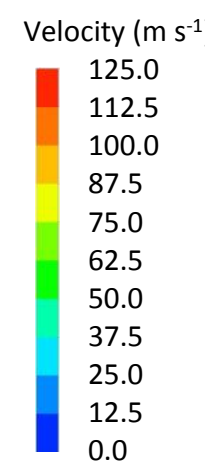

(a)

(b)

(c)

Fig. 13. Longitudinal streamlines of the velocity magnitude at various platoon populations: (a) 2-car platoon (b) 3-car platoon, and (c) 4-car platoon

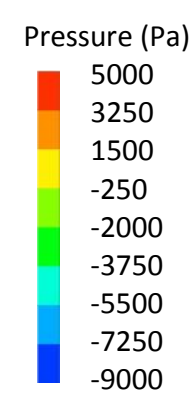

(a)

(b)

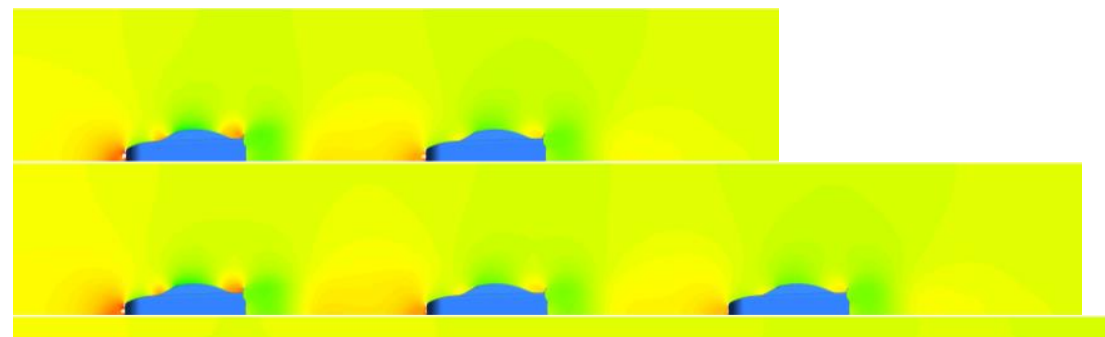

(c)

Fig. 14. Contour plots of the pressure magnitude at various platoon populations: (a) 2-car platoon, (b) 3car platoon, and (c) 4-car platoon

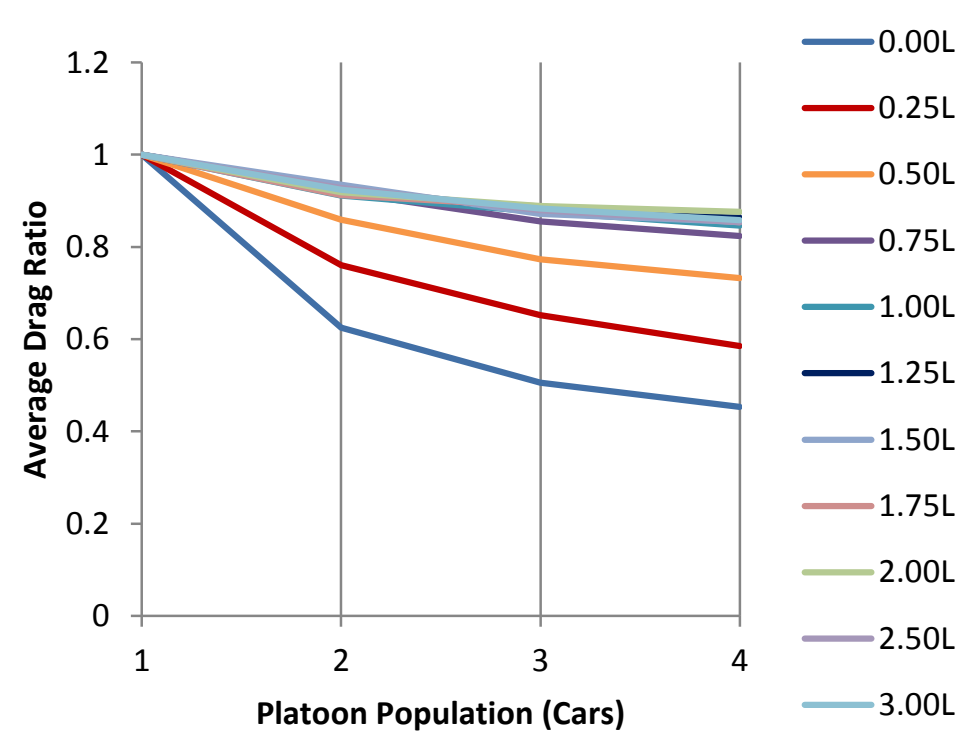

Fig. 15. Average drag ratio against platoon population at various vehicle spacing

\subsection{Side Drafting}

In the study of side drafting, the longitudinal position, $x / L$ and the lateral separation, $y / W$ between two vehicles are correlated with potential overtaking capabilities, where $x$ denotes the streamwise longitudinal position of the trailing car, $y$ denotes the vehicle lateral separation in the $y$ - 
axis, whereas $L$ and $W$ denote the length and width of one car respectively. The schematic diagram of the side drafting configuration is shown in Figure 16.

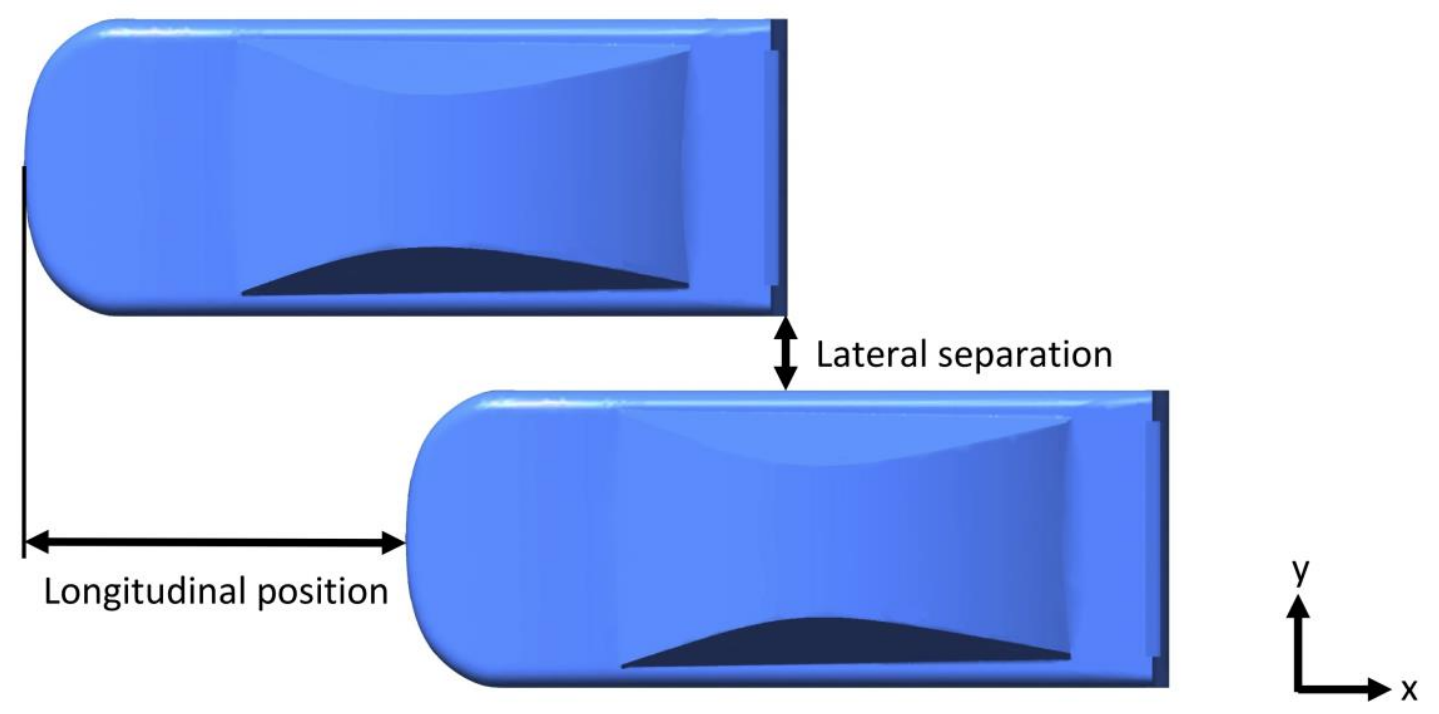

Fig. 16. Side drafting configuration in plan view

Figure 17 exhibits a region of notably higher pressure on the spoiler of the leading car corresponding to where air is displaced onto from the frontal area of the trailing car. The streamline plot also illustrates air velocity increasing significantly before reaching the spoiler of the leading car. A clearer view of this air displacement can be observed in the streamline plot in Figure 18.

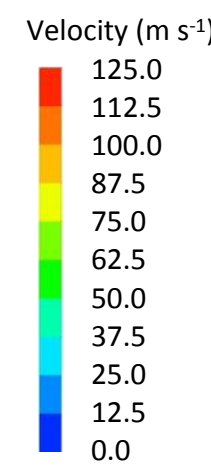

Pressure $(\mathrm{Pa})$
5000
3250
1500
$-250$
$-2000$
$-3750$
$-5500$
$-7250$
$-9000$

(a)

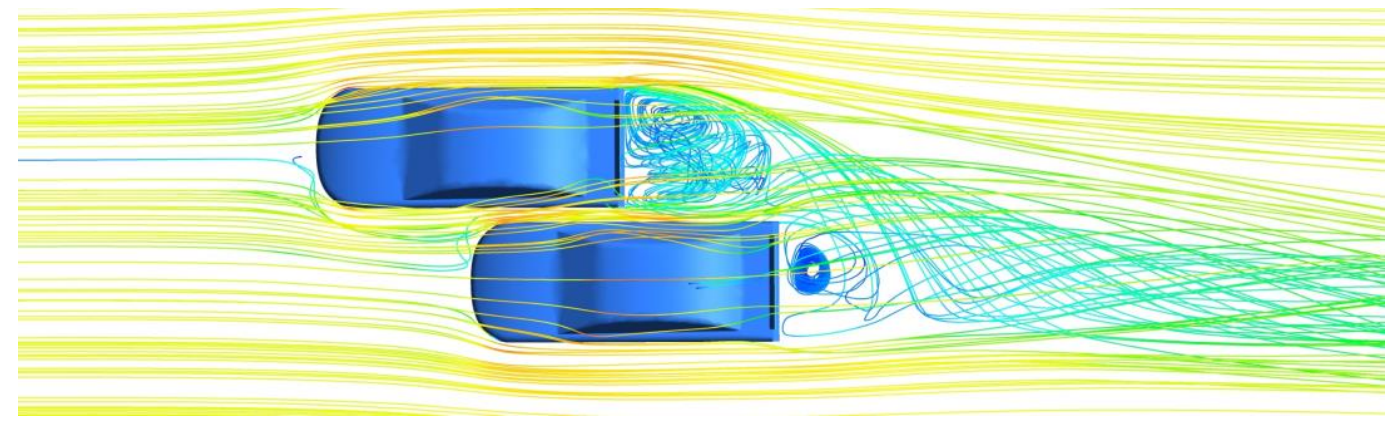

(b)

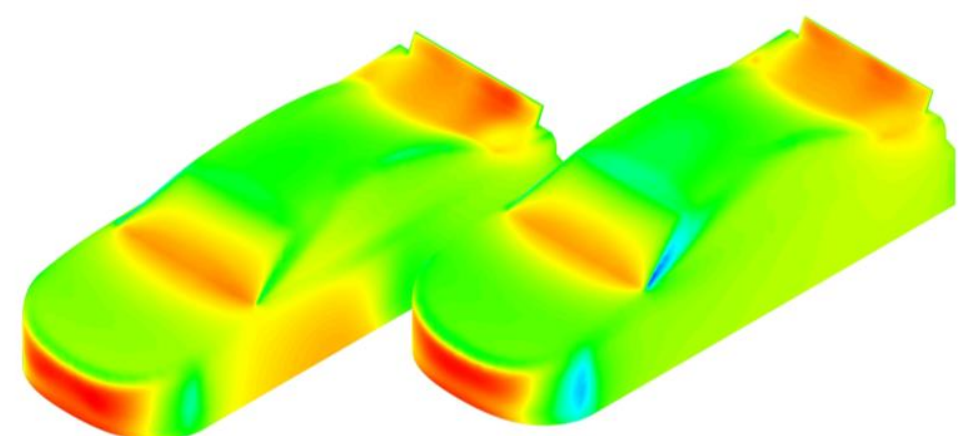

Fig. 17. Side drafting at longitudinal position, $x / L=0.5$ and lateral separation, $y / W=0.125$ car widths: (a) Streamlines of the velocity magnitude, and (b) Contour plot of the pressure magnitude 


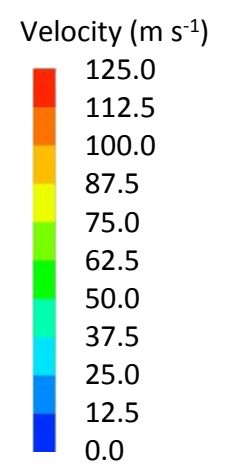

Fig. 18. Streamline plot exhibiting air being displaced from the frontal area of the trailing car towards the spoiler area of the leading car

Figure 19 presents drag ratios of the leading and trailing cars at various longitudinal positions and lateral separations, whereas Figure 20 presents the difference in drag forces experienced between the leading and trailing cars. Drag difference between both cars can be regarded as proportional to the effectiveness of side drafting, where higher drag difference is equivalent to higher potential overtaking capabilities.

From the plot in Figure 19, it is evident that the trailing car in the side drafting configuration with the leading car induces the latter into experiencing huge drag increments as compared to it driving in isolation. Interestingly, the trailing car benefits aerodynamically with some drag reduction as though it is driving in the slipstreaming configuration and is shown to have some air blockage from the leading car in Figure 17. Taking drag differences into consideration, Figure 20 reveals that side drafting at longitudinal positions between $x / L=0.50$ and $x / L=0.75$ are more effective. On the other hand, the ideal range for lateral separation is $y / W \leq 0.125$. Overlapping these two variables reveals the best side drafting configuration to be at $x / L=0.50$ and $y / W=0.125$, where the leading car experiences the highest drag increment and both vehicles yield a maximum drag difference, thus providing the trailing car with the highest aerodynamic advantage against the leading car for maximum overtaking capabilities.

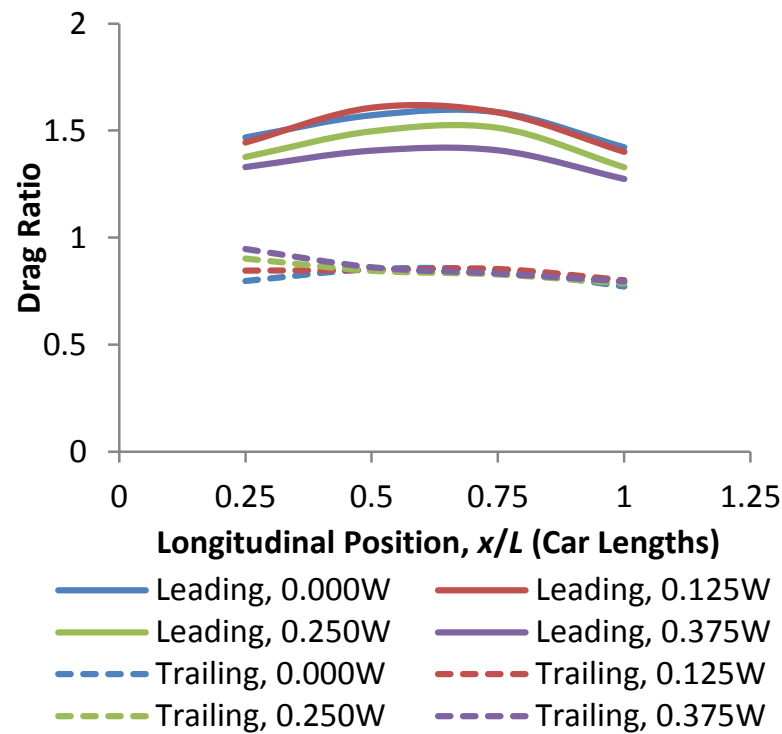

Fig. 19. Drag ratio with respect to longitudinal position for various lateral separations. $\mathrm{W}=\mathrm{Car}$ Width

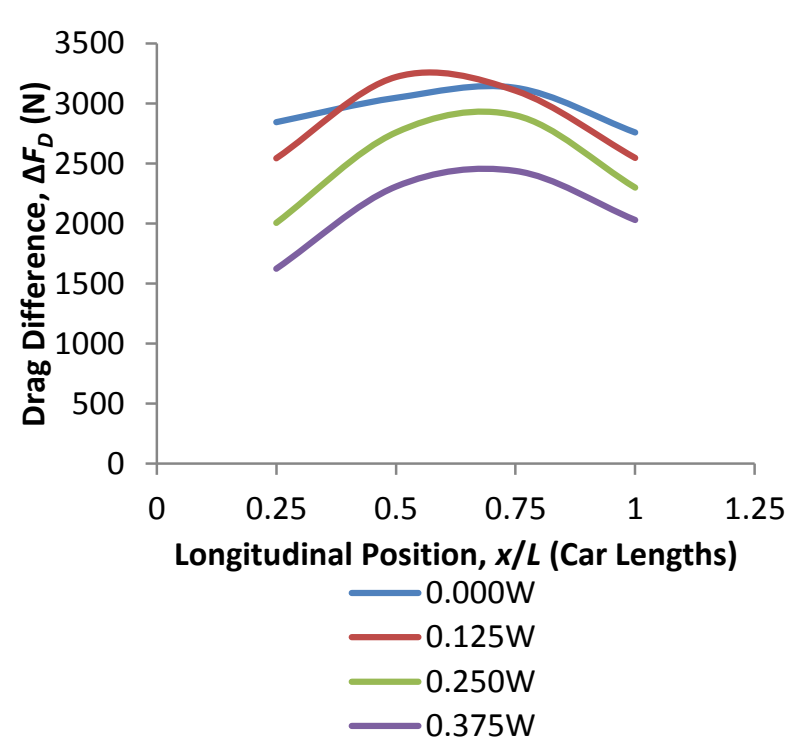

Fig. 20. Drag difference between leading and trailing cars with respect to various longitudinal positions for various lateral separations. $\mathrm{W}=\mathrm{Car}$ Width 


\section{Conclusions}

A comprehensive study of the effects of slipstreaming was performed by varying the vehicle spacing and the platoon population. A thorough investigation of the effects of side drafting was then performed by varying the longitudinal position and lateral separation between the trailing and leading cars. Subsequently, the best racing strategies based on car placements were obtained in both slipstreaming and side drafting configurations with regard to potential drag reduction and overtaking capabilities respectively.

The results prove that slipstreaming is beneficial to platoon members in significantly reducing their aerodynamic drag. The following conclusions are drawn from the slipstreaming investigation:

I. Slipstreaming is most beneficial to trailing cars at short vehicle spacings, $x / L \leq 0.25$.

II. Within the approximate range of vehicle spacings, $x / L=0.25$ to $x / L=1.5$, all trailing members have significant drag increments, with every consecutive downstream member having lesser drag variations. This 'drag bubble' is aerodynamically advantageous for the leading car where it experiences a reduction in drag and has relatively lesser drag than its trailing member.

III. Members at the middle of the platoon generally experience the lowest drag at vehicle spacings, $x / L \leq 1.5$. At larger vehicle spacings, last trailing members experience the lowest drag, followed by every consecutive upstream member.

IV. In considering the average drag of platoon configurations, slipstreaming is significantly beneficial to platoons at vehicle spacings, $x / L<1$. While slipstreaming at vehicle spacings, $x / L>1$ may still somewhat benefit platoons, it does so very ineffectively.

V. Increasing the platoon population decreases the average drag values of the platoon members to only a certain extent. As the platoon gets longer, the drag reductions are not as significant as when the platoon initially had fewer cars.

In the side drafting study, it is found that side drafting is enormously beneficial for trailing cars in raising their overtaking capabilities. Simulating two cars in the side drafting configuration has revealed that the leading car undergoes significant drag increments while the trailing car experiences some drag reduction as in the slipstreaming configuration. The following conclusions are drawn from the findings in the side drafting investigation:

I. Ideal range for longitudinal position is between $x / L=0.50$ and $x / L=0.75$, while the ideal range for lateral separation is $y / W \leq 0.125$. Overlapping these variables reveal that the best side drafting configuration to be at $x / L=0.50$ and $y / W=0.125$.

\section{Acknowledgement}

This research was not funded by any grant.

\section{References}

[1] Zabat, Michael, Nick Stabile, Stefano Frascaroli, and Fred Browand. "Drag forces experienced by 2, 3 and 4-vehicle platoons at close spacings." SAE transactions (1995): 1173-1181. https://doi.org/10.4271/950632

[2] Robertson, Francis H., Frederick Bourriez, Mingzhe He, David Soper, Chris Baker, Hassan Hemida, and Mark Sterling. "An experimental investigation of the aerodynamic flows created by lorries travelling in a long platoon." Journal of Wind Engineering and Industrial Aerodynamics 193 (2019): 103966.

https://doi.org/10.1016/i.jweia.2019.103966

[3] Michaelian, Mark, and Fred Browand. "Quantifying platoon fuel savings: 1999 field experiments." SAE transactions (2001): 1401-1410. https://doi.org/10.4271/2001-01-1268

[4] Hong, Patrick, Bogdan Marcu, Fred Browand, and Aaron Tucker. "Drag forces experienced by two, full-scale vehicles 
at close spacing." (1998).

https://doi.org/10.4271/980396

[5] Brzustowicz, John P., Todd H. Lounsberry, and Jean-Michel Esclafer de La Rode. "Experimental \& Computational simulations utilized during the aerodynamic development of the dodge intrepid $\mathrm{R} / \mathrm{T}$ race car." $S A E$ Transactions (2002): 2387-2403. https://doi.org/10.4271/2002-01-3334

[6] Jacuzzi, Eric, and Kenneth Granlund. "Passive flow control for drag reduction in vehicle platoons." Journal of Wind Engineering and Industrial Aerodynamics 189 (2019): 104-117.

https://doi.org/10.1016/i.jweia.2019.03.001

[7] Belloli, Marco, STEFANO GIUSEPPE Giappino, FABIO CRISTIANO Robustelli, and Claudio Somaschini. "Drafting effect in cycling: Investigation by wind tunnel tests." In 11th conference of the International Sports Engineering Association, ISEA 2016, pp. 38-43. Elsevier Ltd, 2016. https://doi.org/10.1016/i.proeng.2016.06.186

[8] Barry, Nathan, John Sheridan, David Burton, and Nicholas AT Brown. "The effect of spatial position on the aerodynamic interactions between cyclists." Procedia Engineering 72 (2014): 774-779.

https://doi.org/10.1016/j.proeng.2014.06.131

[9] Blocken, Bert, Thijs Defraeye, Erwin Koninckx, Jan Carmeliet, and Peter Hespel. "CFD simulations of the aerodynamic drag of two drafting cyclists." Computers \& Fluids 71 (2013): 435-445.

https://doi.org/10.1016/i.compfluid.2012.11.012

[10] Edwards, Andy G., and William C. Byrnes. "Aerodynamic characteristics as determinants of the drafting effect in cycling." Medicine and science in sports and exercise 39, no. 1 (2007): 170-176. https://doi.org/10.1249/01.mss.0000239400.85955.12

[11] Kyle, Chester R. "Reduction of wind resistance and power output of racing cyclists and runners travelling in groups." Ergonomics 22, no. 4 (1979): 387-397. https://doi.org/10.1080/00140137908924623

[12] Fu, Chen, Mesbah Uddin, and A. Clay Robinson. "Turbulence modeling effects on the CFD predictions of flow over a NASCAR Gen 6 racecar." Journal of Wind Engineering and Industrial Aerodynamics 176 (2018): 98-111. https://doi.org/10.1016/j.jweia.2018.03.016

[13] Ashton, Neil, A. West, S. Lardeau, and A. Revell. "Assessment of RANS and DES methods for realistic automotive models." Computers \& Fluids 128 (2016): 1-15. https://doi.org/10.1016/j.compfluid.2016.01.008

[14] Munson, Bruce R., Theodore H. Okiishi, Wade W. Huebsch, and A. P. Rothmayer. Fluid Mechanics, SI Version. Wiley, 2013.

[15] Tsuei, Lun, and Ö. Savas. "A wind tunnel investigation of the transient aerodynamic effects on a four-car platoon during passing maneuvers." SAE transactions (2000): 1470-1482. https://doi.org/10.4271/2000-01-0875

[16] Hucho, Wolf, and Gino Sovran. "Aerodynamics of road vehicles." Annual review of fluid mechanics 25, no. 1 (1993): 485-537. https://doi.org/10.1146/annurev.fl.25.010193.002413

[17] Choi, Chang-Koon, and Dae-Kun Kwon. "Wind tunnel blockage effects on aerodynamic behavior of bluff body." WIND STRUCT INT J. 1, no. 4 (1998): 351-364. https://doi.org/10.12989/was.1998.1.4.351

[18] Gan, C. J., and S. M. Salim. "Numerical analysis of fluid-structure interaction between wind flow and trees." In Proceedings of the world congress on engineering, vol. 2, pp. 1218-1223. 2014.

[19] Gan, Edward Chern Jinn, and Salim Mohamed Salim. "Numerical Simulation of the Aerodynamic Loads on Trees During Storms." In Transactions on Engineering Technologies, pp. 187-199. Springer, Dordrecht, 2015. https://doi.org/10.1007/978-94-017-9804-4_13

[20] Lanfrit, Marco. "Best practice guidelines for handling Automotive External Aerodynamics with FLUENT." (2005).

[21] ANSYS, Ins. "ANSYS fluent 12.0 User's Guide." New Hampshire: ANSYS INC (2009).

[22] Li, Zhengtong, Jie Xu, Tingzhen Ming, Chong Peng, Jiayue Huang, and Tingrui Gong. "Numerical simulation on the effect of vehicle movement on pollutant dispersion in urban street." Procedia Engineering 205 (2017): 2303-2310. https://doi.org/10.1016/j.proeng.2017.10.104

[23] Shaharuddin, Nur Haziqah, Mohamed Sukri Mat Ali, Shuhaimi Mansor, Sallehuddin Muhamad, Sheikh Ahmad Zaki Shaikh Salim, and Muhammad Usman. "Flow simulations of generic vehicle model SAE type 4 and DrivAer Fastback using OpenFOAM." Journal of Advanced Research in Fluid Mechanics and Thermal Sciences 37, no. 1 (2017): 18-31.

[24] Versteeg, Henk Kaarle, and Weeratunge Malalasekera. An introduction to computational fluid dynamics: the finite volume method. Pearson education, 2007. 
[25] Ahmed, Syed R. "Wake structure of typical automobile shapes." (1981): 162-169. https://doi.org/10.1115/1.3240767

[26] Vino, G., S. Watkins, P. Mousley, J. Watmuff, and S. Prasad. "Flow structures in the near-wake of the Ahmed model." Journal of fluids and structures 20, no. 5 (2005): 673-695. https://doi.org/10.1016/i.jfluidstructs.2005.03.006

[27] Pagliarella, Riccardo M., Simon Watkins, and Angelo Tempia. The effect of rear slant angle on vehicle wakes and implications for platoons. No. 2006-01-0341. SAE Technical Paper, 2006. https://doi.org/10.4271/2006-01-0341 